\title{
中国有机氟化合物卤键研究
}

\author{
刘传志 $a$ 王 辉 $a$ 张丹维*, $a$ 赵 新 $b$ 黎占亭*, $a$ \\ $\left({ }^{a}\right.$ 复旦大学化学系 上海 200438) \\ ( ${ }^{b}$ 中国科学院上海有机化学研究所 上海 200032)
}

\begin{abstract}
摘要 综述了中国学者在有机碘代和溴代氟化物的卤键研究方面的进展. 从 1987 至 1993 年, 陈庆云等发现全氟碘代 烷作为 Lewis 酸可以和胺、醚和六甲基磷酰胺等 Lewis 碱形成供体一受体相互作用, 代表了早期研究现在被称为卤键的 非共价键作用力的重要进展. 2001 年以来, 多个研究组利用卤键开展晶体工程研究. 朱士正等首次证实, 全氟- $\alpha, \varpi$-二 碘烷烃可以和胺、醚和六甲基磷酰胺等通过卤键形成一维扩展阵列结构, 晋卫军等研究了卤键驱动的全氟碘代芳烃与 氮杂芳环的共晶结构, 张丹维和黎占亭等利用卤键诱导产生了双螺旋及四螺旋超分子聚合物. 晋卫军等研究了 $\mathrm{C}$ $\mathrm{I} \cdots \pi$ 卤键在晶体工程中的应用. 利用卤键诱导, 王栋和万立骏等在表面实现了三角型芳香分子共组装形成二维蜂窝型 阵列结构, 王力彦等从两个聚合物或有机分子构筑了单层膜结构和实现了层-层自组装控制. 赵新和黎占亭等合成了 并入三个二氟碘甲基的三臂分子，基于氢键诱导的折叠型受体，建立了溶液相多位点卤键结合的识别模式. 胡文平、龚 和贵、廖良生等利用卤键提高了若干共轭有机分子的不同的材料性能. 多个研究组利用卤键成功控制或提高不同类型 的有机反应的选择性.
\end{abstract}

关键词 卤键; 有机氟化合物; 晶体工程; 分子识别; 自组装; 螺旋体; 人工折叠体; 电子转移反应

\section{Study on Halogen Bonding of Organofluorine Compounds in China}

\author{
Liu, Chuan-Zhi ${ }^{a}$ \\ Wang, Hui ${ }^{a}$ \\ Zhang, Dan-Wei*,a \\ Zhao, $\mathrm{Xin}^{b}$ \\ Li, Zhan-Ting ${ }^{*, a}$ \\ ( ${ }^{a}$ Department of Chemistry, Fudan University, Shanghai 200438) \\ ( ${ }^{b}$ Shanghai Institute of Organic Chemistry, Chinese Academy of Sciences, Shanghai 200032)
}

\begin{abstract}
This review summarizes studies on halogen bonding of fluorine-containing alkyl and aryl iodides in China. From 1987 to 1993, Chen et al. found that there existed donor-acceptor interaction between fluorinated organic (di)iodides, as Lewis acids, and organic Lewis bases such as amines and ethers, which represented early important advances for the research on the non-covalent force currently called as halogen bonding. From 2001 to now, several groups have used halogen bonding as driving force to conduct researches on crystal engineering. In this catogery, Zhu et al. investigated the one-dimensional self-assembly between perfluoro- $\alpha, \varpi$-diioodalkanes and amines, ethers, and hexamethylphosphamide. Jin et al. studied the complexation between fluorinated aryl iodides and various $\mathrm{N}$-heterocycles, whereas Zhang and $\mathrm{Li}$ et al. constructed supramolecular double and quadruple helices from one or two molecular components. Jin et al. conducted extensive studies on $\mathrm{C}-\mathrm{I} \cdots \pi$ halogen bonding and its applications in crystal engineering. Wang and Wan et al. utilized halogen bonding to induce trianglular aromatic molecules to co-assemble into two-dimensional honeycomb arrays on surface, whereas Wang et al. utilized halogen bond to induce mono-layer and layer-by-layer self-assembly of two polymers or organic molecules. Zhao and $\mathrm{Li}$ et al. developed the applications of halogen bonding in solution-phase multi-site molecular recognition of foldamer receptors for $\mathrm{ICF}_{2}$-incorporated tri-armed guests. Hu, Gong, Liao et al. utilized halogen bonding to improve the material properties of a variety of organic aromatic molecules. Several groups have also used halogen bonding to increase the selectivity of a number of organic reactions. Representative examples are described, which highlight the utility of halogen bonding.

Keywords halogen bond; organofluorine compounds; crystal engineering; molecular recognition; self-assembly; helix; foldamer; electron-transfer reaction
\end{abstract}

卤键是卤素原子作为 Lewis 酸的亲电性区域与另外

一个 Lewis 碱分子或离子的亲核性区域之间形成的非共

\footnotetext{
* Corresponding authors. E-mail: zhangdw@fudan.edu.cn; ztli@fudan.edu.cn

Received December 14, 2018; revised December 19, 2018; published online December 21, 2018.

Dedicated to Professor Qingyun Chen on the occasion of his 90th birthday.

Project supported by the National Natural Science Foundation of China (Nos. 21772026, 21432004)

国家自然科学基金(Nos. 21772026, 21432004)资助项目.
} 
价键相互作用. 卤键一定程度上类似于氢键. 在氢键中, 一个氢原子被一个原子、基团或分子提供和接受, 通过 静电吸引而共享. 在卤键中, 有机分子提供一个卤素原 子, 与另一个物种的亲电性区域形成静电吸引实现共 享. 氢键的概念形成于二十世纪一二十年代 ${ }^{[1]}$, 后由 Pauling ${ }^{[2]}$ 命名和推广而被广泛接受. 而早在 1863 年, Guthrie $^{[3]}$ 就观察到碘和氨能形成复合物. 1954 年, Hassel 等 ${ }^{[4]}$ 首次通过晶体 $X$ 衍射实验观察到单质溴和二氧六环 之间存在 $\mathrm{Br} \cdots \mathrm{O}$ 短距离接触 $(2.71 \AA)$, 证实了二者之间 的静电吸引作用. 1978 年, Dumas 等 ${ }^{[5]}$ 首次使用卤键 (halogen bond)描述这一静电相互作用. 1998 年以来, Resnati 和 Metrangolo 等 ${ }^{[6]}$ 利用卤键开展晶体工程方面的 研究, 推动了其在超分子化学和分子自组装领域的应 用. 由于卤素原子电负性普遍较高, 在有机分子中, $\mathrm{C}-$ $\mathrm{X}$ 键极化导致卤素原子带有部分负电荷. 但理论计算表 明 ${ }^{[7]}$, 在有机分子中 $\mathrm{C}-\mathrm{X}$ 键附近引入拉电子基团后, 其碘或澳的外层电子云发生极化, 向 $\mathrm{C}-\mathrm{X}$ 键方向移动, 导致在 C-X键相反一侧形成所谓缺电性的具有表面正 静电势的区域: $\sigma$-洞 $(\sigma$-hole), 从而能作为电子受体形成 卤键. 氟原子具有最高的电负性, 因此有机氟化合物中 碘和溴形成卤键的能力较强. 2013 年, 国际纯粹和应用 化学联合会(IUPAC)建议把卤键定义为: “当有证据表明, 在一个分子实体的卤原子的亲电区域和另外一个或同 一个分子实体的亲核区域存在净的静电吸引作用时, 可 认为形成了卤键” ${ }^{[8]}$. 图 1 给出了形成卤键的典型供体 和受体结构原子.

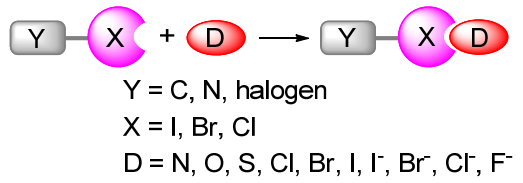

图 1 卤键形成示意图

Figure 1 Schematic representation for the formation of halogen bonds

1965 年, Pullin 等 ${ }^{[9]}$ 通过相图研究提出, 全氟碘代烷 和胺形成电荷转移络合物 (charge-transfer complex). 1978 年, Gutman 等 ${ }^{[10]}$ 发现, 这一络合现象存在于全氟碘 代烷和许多 Lewis 碱中间. 陈庆云等 ${ }^{[11 ~ 13]}$ 在 20 世纪 80 年代曾系统研究碘代有机氟化合物作为电子受体发生 的电子转移反应, 发现液态的全氟二碘乙烷和已烷可以 与同为液态的吗啡啉、四甲基乙二胺及二氧六环等形成 固体电荷转移络合物. 并揭示 $\mathrm{ICF}_{2}$ 的 ${ }^{19} \mathrm{~F} N \mathrm{NMR}$ 化学位 移变化与溶剂的供体数 (donor number, DN) 参数存在着 线性关系 ${ }^{[13]}$. 陈庆云等 ${ }^{[14]}$ 还发现全氟二碘烷烃与 $N, N, N^{\prime} N^{\prime}$-四甲基-对-苯二胺也可以形成熔点高于后者 的电荷转移络合物, 并由此促进二者发生光诱导的电子
转移反应. 这些研究为卤键作为一种新的非共价键作用 力的确立提供了早期的重要实验基础.

卤键作为一种非共价键作用力, 在晶体工程领域获 得广泛的应用 ${ }^{[15]}$. 近年来在新的光电材料设计 ${ }^{[16]}$, 生物 活性分子设计及药物发现及控制反应选择性等方面也 取得了许多重要进展 ${ }^{[17 \sim 19]}$. 国内学者在涉及非氟代分

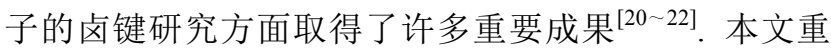
点介绍国内学者在有机氟化合物的卤键研究方面取得 的进展.

\section{1 晶体工程}

晶体工程研究可揭示分子间相互作用的机制和规 律, 研究分子固态有序结构及其性质. 通过超分子合成 子和各种二级结构单元设计, 化学家可以构造许多形式 的复杂结构, 很多结构具有独特的性质或功能. 氢键和 配位作用是开展晶体工程研究的常用非共价键作用

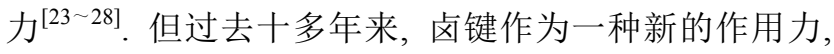
也广泛应用于晶体工程研究 ${ }^{[29]}$. 卤键具有显著的正交 性和方向性 ${ }^{[30]}$, 在与其它非共价键作用力共同驱动复 杂有序结构的构建方面具有独特优势.

\section{1 基于全氟- $\alpha, \varpi$-二磑代烷烃合成子的晶体工程}

由于氟原子的拉电子效应，全氟碘代烷的碘原子具 有显著的 $\sigma$-洞, 是一类重要的卤键供体. 2001 年朱士正 等 ${ }^{[31]}$ 报道了全氟代- $\alpha, \varpi-$ 二碘乙烷、丁烷和己烷与二氧六 环和六甲基磷酰胺的 $1: 1$ 络合物的晶体结构(图 2). 前 一系列结构中，每个二碘烷烃的一个碘原子与二氧六环 的一个氧原子形成 $\mathrm{I} \cdots \mathrm{O}$ 卤键, 二者距离显著小于两个 原子的范德华半径之和. 而一个六甲基磷酰胺的氧原子 和两个氟代烷烃的一个碘原子形成分叉型卤键. 两个系 列都通过 $\mathrm{I} \cdots \mathrm{O}$ 卤键形成一维阵列结构. 二氧六环与单 质澳也可以形成类似的一维阵列 ${ }^{[4]} .1987$ 年陈庆云等 ${ }^{[2]}$ 也曾报道四氟二碘乙烷与二氧六环形成结晶型固体, 并 提出了相应的 $\mathrm{I} \cdots \mathrm{O}$ 相互作用. 这一研究确认了在固体 中, 二者形成了一维扩展结构, 而不是 $1+1$ 的环型结 构, 并发现四氟二碘乙烷与 1,4-二甲基哌嗪、 $N$-甲基吗 啡啉、吡嗪、2-甲基和 2-乙基吡啶及四甲基乙二胺等形 成卤键 ${ }^{[32]}$.

\section{2 基于全氟磑代苯合成子的晶体工程}

四氟-对-二碘苯 $(3)$ 的两个碘原子反向排列, 是形 成一维卤键阵列结构的理想单体. 吉宝明等 ${ }^{[33]}$ 曾研究 了其和 $4 a \sim 4 c$ 之间的相互作用(图 3). $4 a \sim 4 c$ 的羰基氧 原子和杂环氮原子都是较强的卤键受体. 在晶体结构 中, 3 和 $4 \mathrm{a}$ 扭曲排列, 3 的两个碘原子与 $4 \mathrm{a}$ 的氧原子和 一个氮原子形成折叠型线性卤键阵列, $\mathbf{4 a}$ 的另外一个 


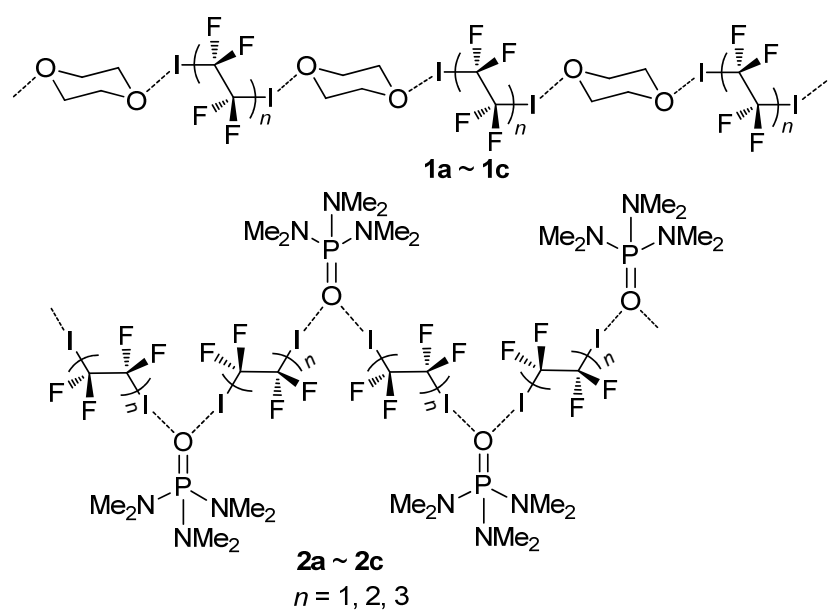

图 2 全氟二碘 $\alpha, \varpi$-烷烃与二氧六环和六甲基磷酰胺形成的 一维卤键阵列

Figure 2 Halogen bonded arrays formed by perfluoro- $\alpha, \varpi-$ diiodoalkanes, dioxane or hexamethylphosphoramide

氮原子没有介入卤键. 而 $4 \mathrm{~b}$ 和 $4 \mathrm{c}$ 都形成两种不同的分 叉型卤键. $4 \mathrm{a}$ 的两个氮原子不能形成分叉型卤键的一个 可能原因是, 分叉型卤键要求两个分子对称排列, 将导 致羰基氧形成的另一个卤键形成 $180^{\circ}$ 夹角. 而羰基氧的 孤对电子取向使得其倾向于在 $120^{\circ}$ 方向形成分子间静 电吸引作用. 相反, 化合物 $\mathbf{4 b}$ 和 $4 \mathrm{c}$ 分子两侧都能形成 分叉型 $\mathrm{I} \cdots \mathrm{N}$ 或 $\mathrm{I} \cdots \mathrm{O}$ 卤键，其与两个分子的氧和氮的孤 対电子取向一致.<smiles></smiles>

3

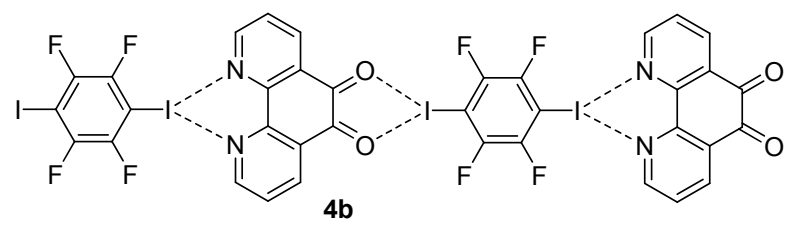

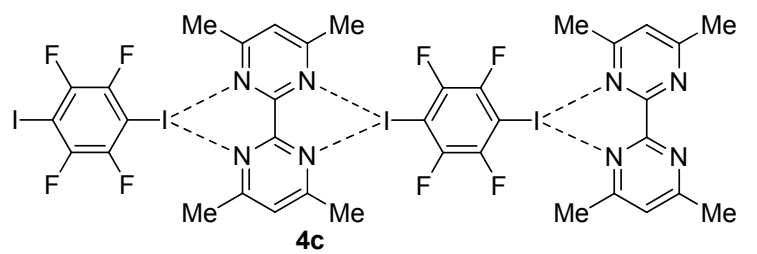

图 3 化合物 3 与 $4 a \sim 4 c$ 形成的共晶卤键模式

Figure 3 Discrete halogen bonding patterns formed between 3 and $\mathbf{4 a} \sim \mathbf{4} \mathbf{c}$ in co-crystals

间东鹏等 ${ }^{\left[{ }^{[34}\right.}$ 就卤键驱动的有机分子共晶发光开展 了系列研究. 发现 3 与二㲵基化合物 $\mathbf{5}$ 形成一维卤键阵 列结构(图 4) ${ }^{[34 a]}$. 与微米尺度的类似物相比, 这一卤键
共晶展示出独特的单光子和双光子磷光和荧光发射. 加 热导致化合物 3 挥发, 相应的晶体产生发光红移, 从蓝 绿色变为黄色. 这一卤键促进的发光现象可以扩展到其 它不同的结构体系 ${ }^{[34]}$.
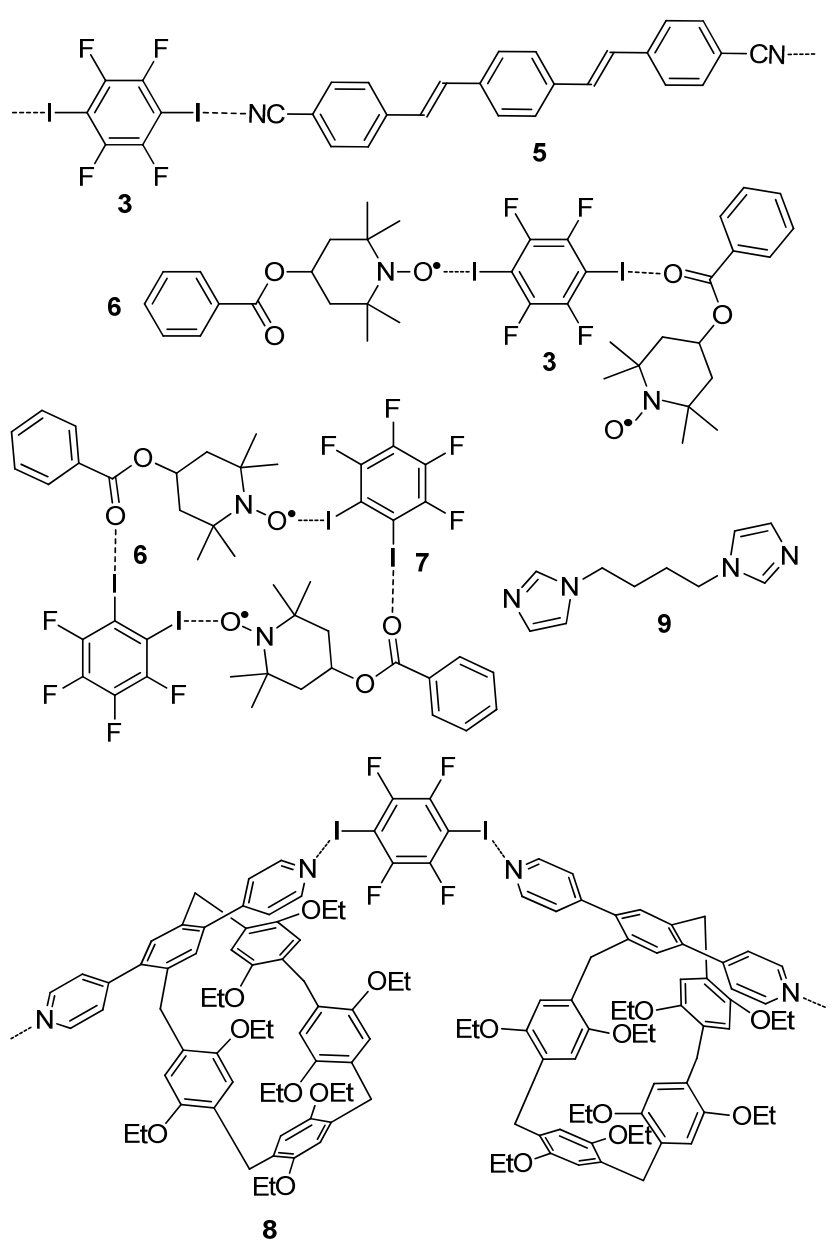

图 4 二碘化合物 3 或 7 与 $5 、 6$ 或 8 形成的共晶卤键模式及 化合物 9 的结构

Figure 4 Discrete halogen bonding patterns formed between 3 or 7 and 4,6 , or 8 and the structure of compound 9

晋卫军等 ${ }^{[35]}$ 报道了化合物 $\mathbf{3}$ 与 $\mathbf{6}$ 的四甲基哌嗪 $\mathrm{N}$ $\mathrm{O}$ 自由基氧原子形成两个 $\mathrm{I} \cdots \mathrm{O}$ 卤键(图 4), 而 6 的 $\mathrm{N}-$ $O$ 自由基氧原子和羰基氧原子皆可以与四氟-邻-二碘 苯(7)形成 $\mathrm{I} \cdots \mathrm{O}$ 卤键, 并形成一个 $2+2$ 的超分子大环. 黄飞鹤等 ${ }^{[36]}$ 合成了柱[5]芳烃双吡啶衍生物 8 , 其与 $\mathbf{3}$ 也 可以通过 $\mathrm{I} \cdots \mathrm{N}$ 卤键形成一维聚合物阵列，而柱[5]芳烃 内腔包结正己烷, 形成独特的拟轮烷结构(图 4). 韩成友 等 ${ }^{[37]}$ 还发现, 柱 [5]芳烃醚氧原子可以作为卤键受体与 $\mathbf{3}$ 形成三维超分子共晶结构, 柱芳烃对双咪唑分子 9 的包 结作用能够促进共晶结构.

全氟碘代化合物还可以通过 $\mathrm{C}-\mathrm{I} \cdots \pi$ 卤键控制两个 分子在共晶中有序排列 ${ }^{[38]}$. 晋卫军等 ${ }^{[39]}$ 利用这一弱的 卤键模式开展了系列晶体工程研究. 2012 年他们 ${ }^{[39 a]}$ 报 
道, 化合物 $\mathbf{3}$ 与芴 $\mathbf{1 0}$ 形成共晶(图 5), 驱动力来自于分子 间 $\mathrm{C}-\mathrm{I} \cdots \pi$ 卤键和 $\mathrm{C}\left(\mathrm{sp}^{3}\right)-\mathrm{H} \cdots \pi$ 氢键. 联苯、芸、菲及 1-氮杂葱等也可以与 $\mathbf{3}$ 形成这一弱的卤键模式 ${ }^{[39 b, 39 c]}$.

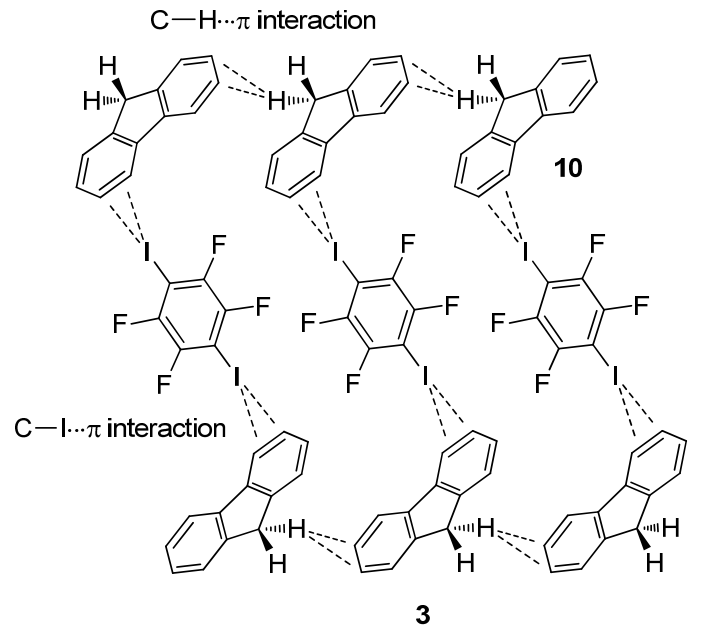

图 5 二碘化合物 3 与芴 10 形成的共晶的 $\mathrm{C}-\mathrm{I} \cdots \pi$ 卤键和 $\mathrm{C}\left(\mathrm{sp}^{3}\right)-\mathrm{H} \cdots \pi$ 氢键模式

Figure $5 \mathrm{C}-\mathrm{I} \cdots \pi$ halogen bonding and $\mathrm{C}\left(\mathrm{sp}^{3}\right)-\mathrm{H} \cdots \pi$ hydrogen bonding patterns formed between $\mathbf{3}$ and $\mathbf{1 0}$ in co-crystal

张丹维和黎占亭等 ${ }^{[40]}$ 合成了化合物 $11 \sim 13$ (图 6), 在其两端分别引入卤键供体 $\mathrm{CF}_{2} \mathrm{I}$ 或氟代碘苯和吡啶作 为卤键受体. 晶体结构显示, 分子间的 $\mathrm{I} \cdot \mathrm{N}$ 卤键诱导这 些分子形成单组分的 P和 M 手性超分子螺旋结构. 这些 单螺旋结构可以堆积形成新的超分子双螺旋结构. 化合 物 13 形成的双螺旋结构进一步堆积形成类似 G-四联体 的超分子四螺旋结构. 端位并入两个吡啶的对称分子 14 与 $1 a$ 通过 N $\cdots$ I 卤键诱导形成双组分交替排列的 P 和 $M$ 超分子螺旋体, 一轮螺旋有四个分子 $(2+2)$ 组成. 两个 $\mathrm{P}$ 螺旋结构和两个 $\mathrm{M}$ 螺旋结构交替堆积, 形成另一 个卤键超分子四螺旋结构 (图 6). 这一四螺旋结构在二 维空间进一步堆积形成平面阵列结构. 分子内氢键和分 子间卤键展示出高度的正交性. 异丁基的引入产生较大 的位阻, 可能有利于形成大的螺距, 也有利于超分子四 螺旋结构的形成.

\section{2 表面和界面自组装}

以氢键为驱动力在表面和界面形成有序单层或膜 结构, 文献已有大量报道 ${ }^{[41,42]}$. 王栋和万立骏等 ${ }^{[43]}$ 报道 (图 7), 在扫描隧道显微镜针尖电刺激下, 三位点化合物 15 和 16 在石墨表面可以通过分子间端位 $\mathrm{I} \cdots \mathrm{N}$ 卤键诱 导, 形成蜂窝型二维阵列结构, 而 $\mathbf{1 5}$ 与 $\mathbf{1 7}$ 在类似卤键 和 $\pi-\pi$ 堆积作用驱动下, 可以形成二维菱形超分子结构. 当使用 3 为卤键供体时, 可以形成空穴更大的蜂窝型二 维结构. 单体刚性结构加上卤键的高度方向性, 使得在

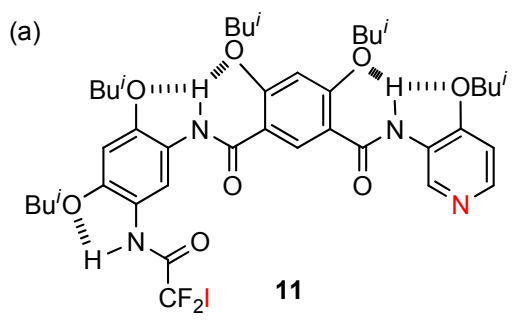

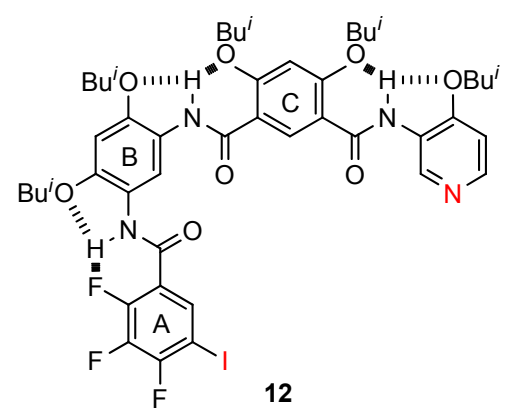

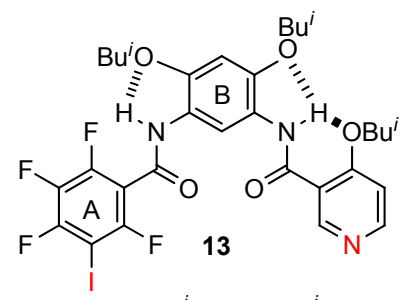

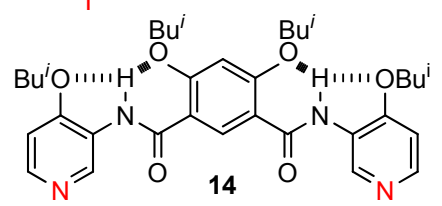

b)
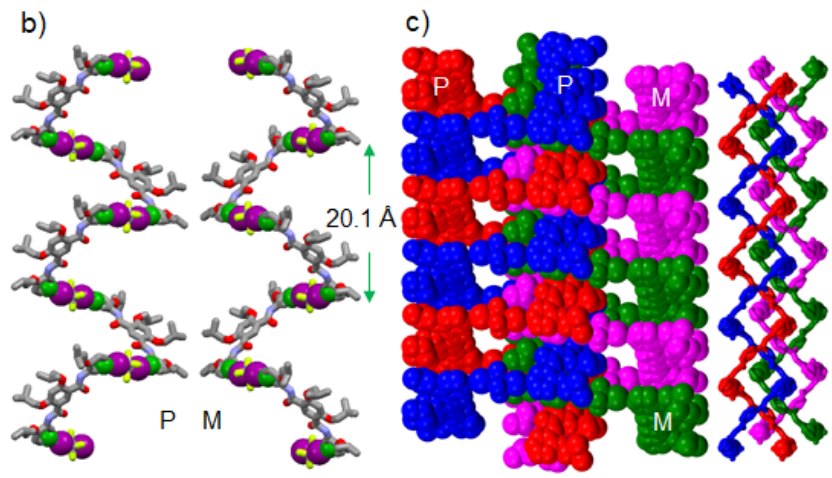

图 6 (a)化合物 11 14 的结构, (b) $1 \mathrm{a}$ 和 14 形成的 P 和 M 型 手性超分子单螺旋体, (c) P, P 和 M, M 超分子四螺旋体

Figure 6 (a) Structure of compounds 11 14, (b) P and M supramolecular single helices formed by $\mathbf{1 a}$ and 14, and (c) P, P and M, M supramolecular quadruple helix

Left: view from the opposite direction of the $a$ axle, right: view from the $c$ axle

大面积纳米尺度实现两个分子的有序排列.

王力彦等 ${ }^{[44 a]}$ 报道, 化合物 18 和 19 通过 N …I 卤键 在表面形成鱼骨型的二维超分子阵列(图 8), 而聚合物 P20 和 P21 通过 $\mathrm{I} \cdots \mathrm{N}$ 卤键诱导层-层自组装, 从而形成 多层膜结构 ${ }^{[44 b]}$. 两个聚合物之间形成的卤键得到 $\mathrm{X}$ 射 线光电子能谱证实. 多层膜表面呈平面排列, 厚度约 

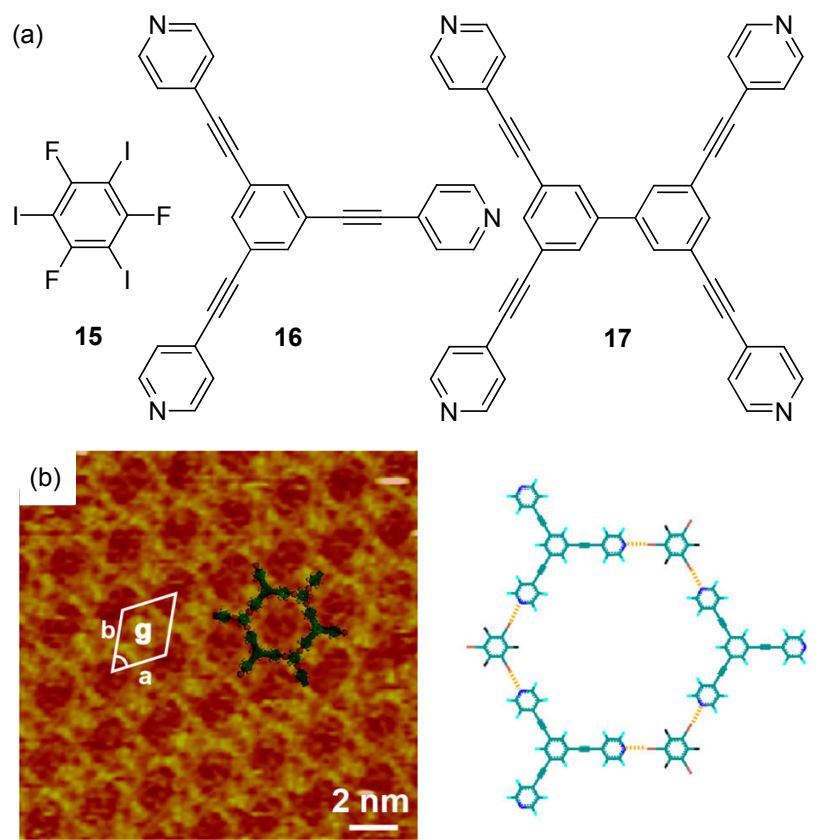

(c)
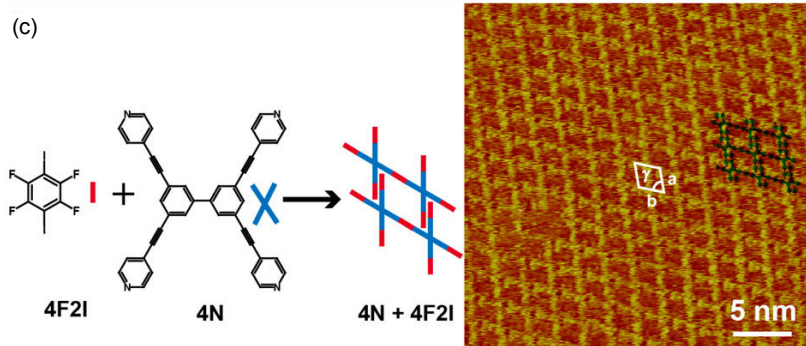

图 7 (a)化合物 15 17 的结构、(b) 15 和 16 形成的二维蜂窝 型结构、 (c) 15 和 17 形成的二维菱形阵列结构

Figure 7 (a) Structure of compounds $15 \sim 17$, (b) 2D honeycomb supramolecular structure formed by $\mathbf{1 5}$ and $\mathbf{1 6}$, and (c) 2D rhombic structure formed by $\mathbf{1 5}$ and $\mathbf{1 7}$

$1.3 \mathrm{~nm}$. 在甲醇中, 这一卤键驱动的分子膜稳定性与氢 键驱动的类似结构相当. 这类聚合物之间形成的卤键应 具有协同加和性, 是相应氢键和配位作用驱动的聚合物 自组装概念的扩展.

\section{3 溶液相分子识别一卤键多价性}

在溶液中, 单一甾键由于结合强度低一般难以定量 研究 ${ }^{[45]}$. 黎占亭等 ${ }^{[46 a]}$ 曾确立了 1,4 -二苯基三氮唑 $\mathrm{C}^{5}-\mathrm{H}$ 的分子内三中心氢键模式. 利用此分子内氢键, 赵新和 黎占亭等 ${ }^{[46 b, 46 c]}$ 构筑了一系列包括 22 的三氮唑折叠或螺 旋结构. 在二氯甲烷等弱极性有机溶剂中, 其通过多个 $\mathrm{N}^{2} \cdots \mathrm{I}$ 卤键, 对 $23 \mathrm{c}$ 及 24 等三臂分子展示出很强的结合 (图 9), 与 23c 形成的 $1: 1$ 的络合物结合常数达到

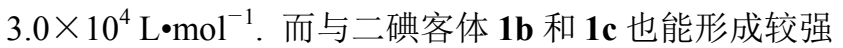
的卤键结合. 化合物 22 与 $23 \mathrm{a}$ 和 $23 \mathrm{~b}$ 等也能通过协同作 用形成 $\mathrm{N}^{2} \cdots \mathrm{Cl}$ 和 $\mathrm{N}^{2} \cdots \mathrm{Br}$ 卤键, 形成较为稳定的 $1: 1$ 络合物. 张丹维和黎占亭等 ${ }^{[46 \mathrm{~d}]}$ 进一步合成了类似骨架 (a)

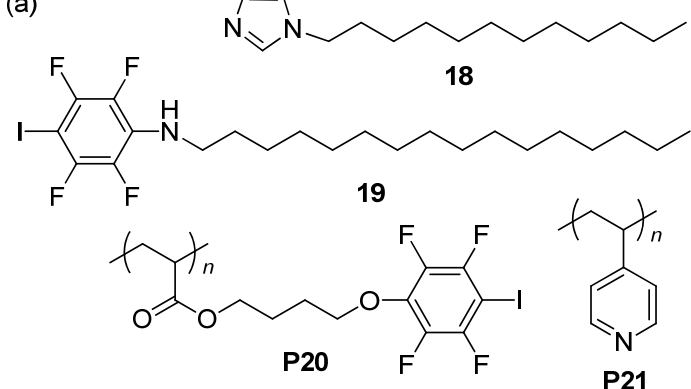

(b)

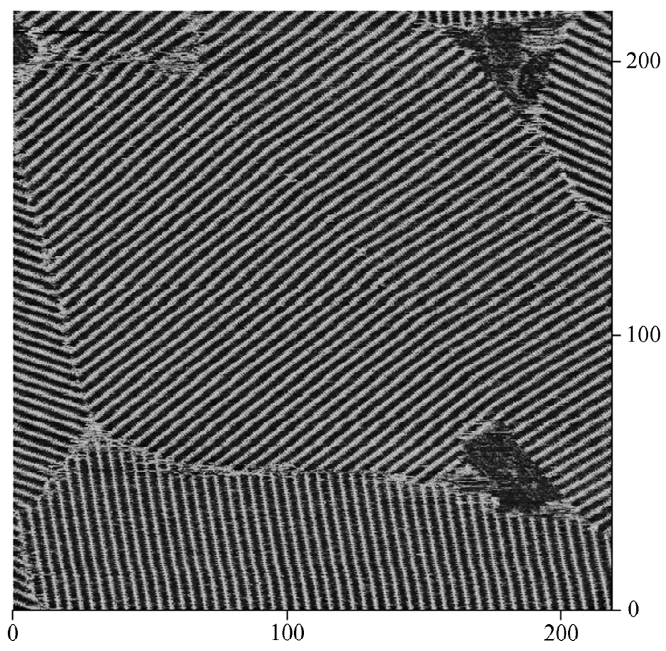

图 8 (a)化合物 18 和 19 及聚合物 P20 和 P21 的结构以及(b) 18 和 19 形成的卤键络合物的扫描隧道显微镜图像

Figure 8 (a) Structure of compounds 18 and 19 and polymers $\mathbf{P 2 0}$ and P21, and (b) the scanning tunneling microscopic image of the halogen bonding complex

的三氮唑大环，发现相应大环能够与 $23 \mathrm{c}$ 等形成更高稳 定的卤键络合物.

\section{4 卤键促进的有机材料}

晋卫军等报道 ${ }^{[47 \mathrm{a}]}$, 化合物 3 与咔唑通过 $\mathrm{C}-\mathrm{I} \cdots \pi$ 卤 键及 $\mathrm{C}-\mathrm{H} \cdots \mathrm{I}$ 和 $\mathrm{C}-\mathrm{H} \cdots \mathrm{F}$ 氢键形成 $2: 1$ 共晶, 并通过 自旋轨道耦合显著促进咔唑的磷光. 理论计算表明, $\mathrm{C}$ - $\mathrm{I} \cdots \pi$ 卤键键能约为 $23.0 \mathrm{~kJ} \bullet \mathrm{mol}^{-1}$, 表明这一卤键较 为稳定. 他们还发现 ${ }^{[47 b], 3}$ 和 4-苯基吡啶氮氧化物 25 通 过分叉型 $\mathrm{I} \cdots \mathrm{O}$ 卤键和 $\mathrm{C}-\mathrm{H} \cdots \mathrm{F}$ 氢键形成 $2+2$ 型空穴 (图 10), 这一空穴可以通过 $\pi-\pi$ 堆积和其它弱分子间氢 键包结咔唑、菜、菲和 1-氮杂菲等. 这一内穴可以调节 其大小和形状，以适应包结不同的客体. 在室温下包结 客体的共晶可以发射不同波长的可见光. 通过选择不同 的卤键受体和包结的客体 ${ }^{[47 \mathrm{c}]}$ ，可以控制这一基于化合 物 3 形成的卤键共晶的发光波长.

王明亮等 ${ }^{[48]}$ 报道，化合物 $\mathbf{3}$ 和 $\mathbf{2 6 a} \sim \mathbf{2 6}$ d 都可以通 过 $\mathrm{I} \cdots \mathrm{O}(=\mathrm{C})$ 卤键和 $\mathrm{C}-\mathrm{I} \cdots \pi$ 卤键等形成共晶(图 11). 共 晶使得卤键受体 26a 26d 通过三线态-三线态湮灭过程 产生延迟苂光发射，从而导致荧光寿命延长，表明羰基 


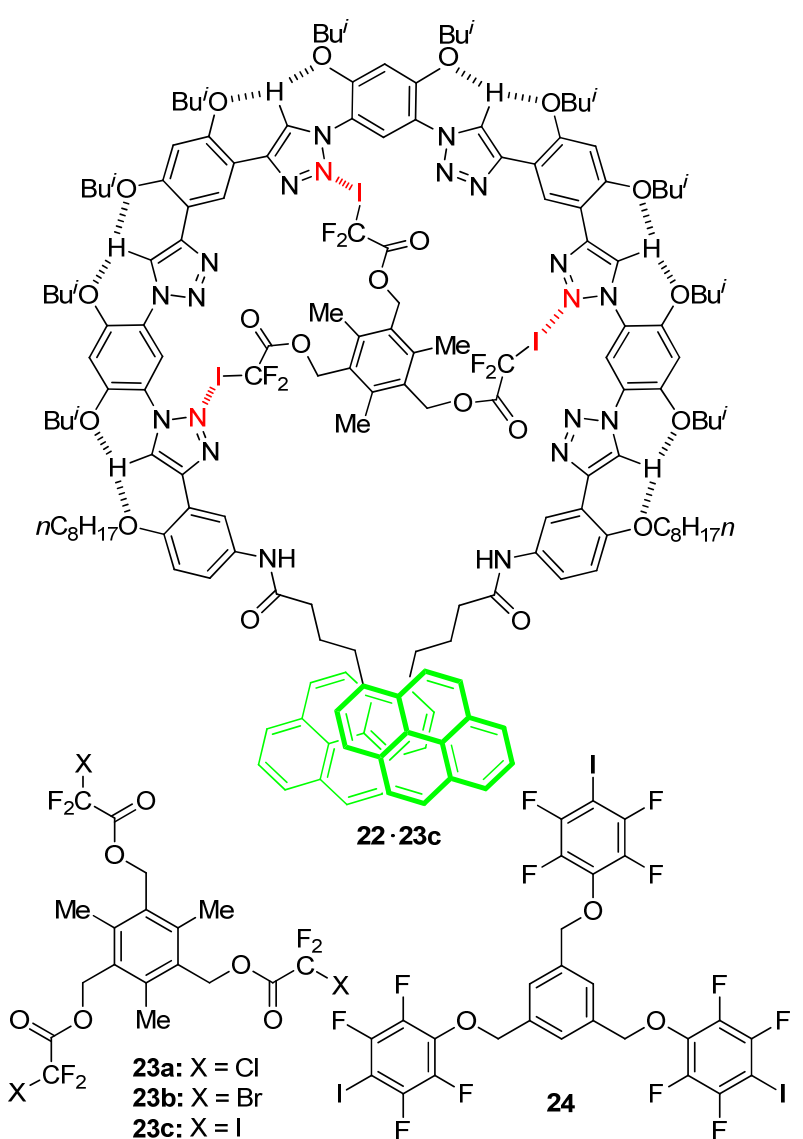

图 9 化合物 $22,23 \mathrm{a} \sim 23 \mathrm{c}$ 和 24 及卤键络合物 $22.23 \mathrm{c}$ 的结构

Figure 9 Structures of compounds 22, 23a $\sim 23 \mathrm{c}$ and 24 and halogen bonded complex 22.23c

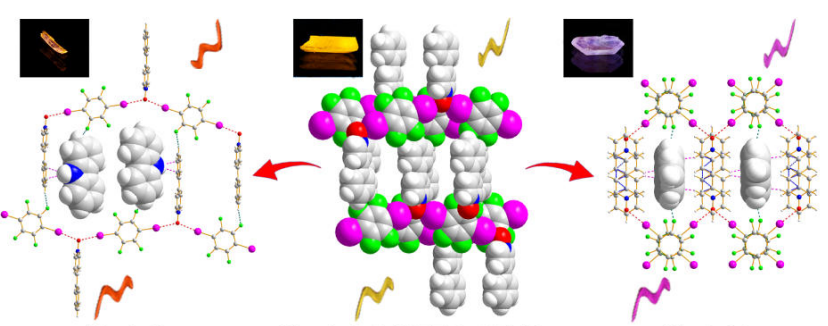

图 10 化合物 3 和 25 通过 $\mathrm{I} \cdots \mathrm{O}$ 卤键和 $\mathrm{C}-\mathrm{H} \cdots \mathrm{F}$ 氢键形成 $2+2$ 空穴(中), 并包结咔唑(左)和萗(右)等

Figure $10 \mathrm{I} \cdots \mathrm{O}$ halogen bonding and $\mathrm{C}-\mathrm{H} \cdots \mathrm{F}$ hydrogen bonding-induced cavity (middle) formed by $\mathbf{3}$ and $\mathbf{2 5}(2+2)$ and its encapsulation for carbazole (left) or naphthalene (right)

氧作为受体形成卤键, 诱导共轭体系电子结构发生变 化.

董焕丽等 ${ }^{[49]}$ 报道, 化合物 $\mathbf{1 5}$ 和 1,2-二(4-吡啶)乙烯 (27)通过分子间 $\mathrm{I} \cdots \mathrm{N}$ 卤键和 $\pi-\pi$ 堆积作用诱导相互堆积, 并产生电荷转移作用, 形成线型晶体(图 12), 构筑了一 类准一维半导体. 这类半导体能发射较强的紫罗兰光, 发光效率达到 $26.1 \%$, 并能限制在一维轴方向传播. 而 27 与 3 形成的方型共晶揭示两个分子交替堆积, 但没有 电荷转移作用发生, 而是形成了有机绝缘体. 这类双分 子绝缘体能展示独特的白光发射和二维光波导性质. 化

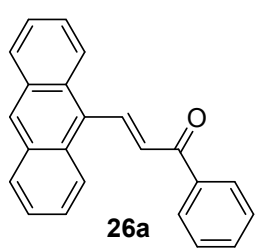<smiles>COc1ccc(C(=O)/C=C/c2ccc3ccc4cccc5ccc2c3c45)cc1</smiles>
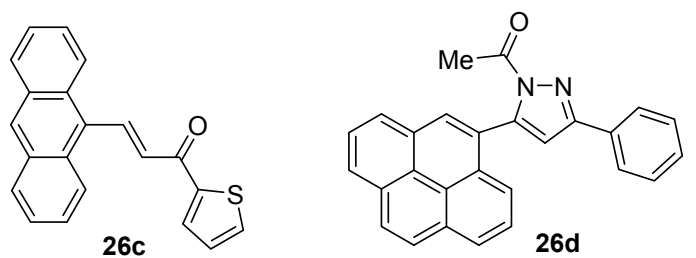

图 11 化合物 26a $\sim 26 d$ 的结构

Figure 11 Structure of compounds 26a $\sim 26 \mathrm{~d}$

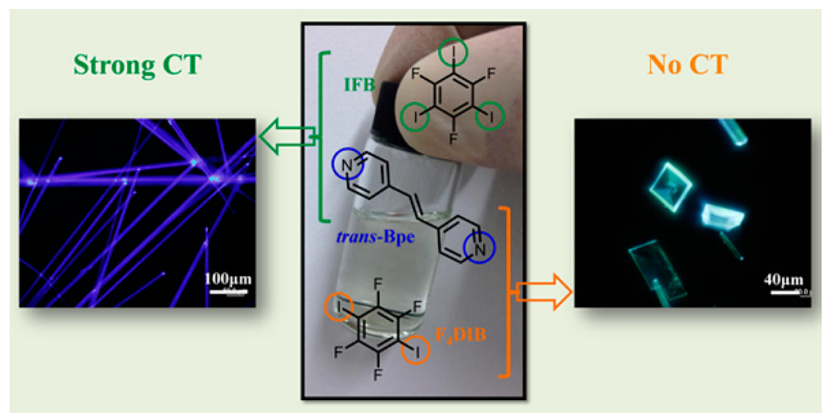

图 12 化合物 27 与 15 和 3 通过卤键和其它相互作用驱动形 成发射紫罗兰光一维纳米线半导体(左)和发射白光方型晶体 绝缘体(右)

Figure 12 1D violet-blue wire semiconductors (left) formed by compounds 27 and 15, and co-crystal white-light-emission insulators (right) formed by 27 and 3

合物 27 和 3 形成共晶后, 其固有光谱态不发生明显变 化, 从而为有机白光材料设计提供了新的设计原理.

通过超分子排列控制有机半导体的微观结构和薄 膜形态对于改进器件性能起到重要作用. 龚和贵和高希 珂等 ${ }^{[50]}$ 合成了化合物 $\mathbf{2 8}$, 发现其与氮杂环 $\mathbf{2 9}$ 和 30 等都 能形成分子间 $\mathrm{I} \cdots \mathrm{N}$ 卤键(图 13). 化合物 $\mathbf{2 8}$ 作为 $\mathrm{n}$-型有 机半导体形成卤键络合物后, 其作为有机场效应晶体管 的器件性能高于纯的化合物 $\mathbf{2 8}$, 平均电子迁移率增加 了 2 倍以上.

有机晶体的各向异性排列有助于提高其光电性质, 而有规则的形貌和光子限域可促进利用二维有机晶体 构筑芯片规模的平面光子系统. 王雪东和廖良生等 ${ }^{[51]}$ 利用化合物 3 和 31 形成 $\mathrm{I} \cdots \mathrm{N}$ 卤键产生共晶, 控制二者 的有序排列(图 14). 这一共晶能展示不对称光波导, 沿 [010]晶体方向及反向的光损耗系数分别为 0.0894 和 $0.036 \mathrm{~dB} \cdot \mu \mathrm{m}^{-1}$. 这一差异被归结于共晶中分子各向异 性堆积模式引起的单向全内折射. 这一晶体内分子方向 性定位实现的不对称光子输送, 可用于制备具有多个输 入和输出通道的新的微型光学逻辑门. 


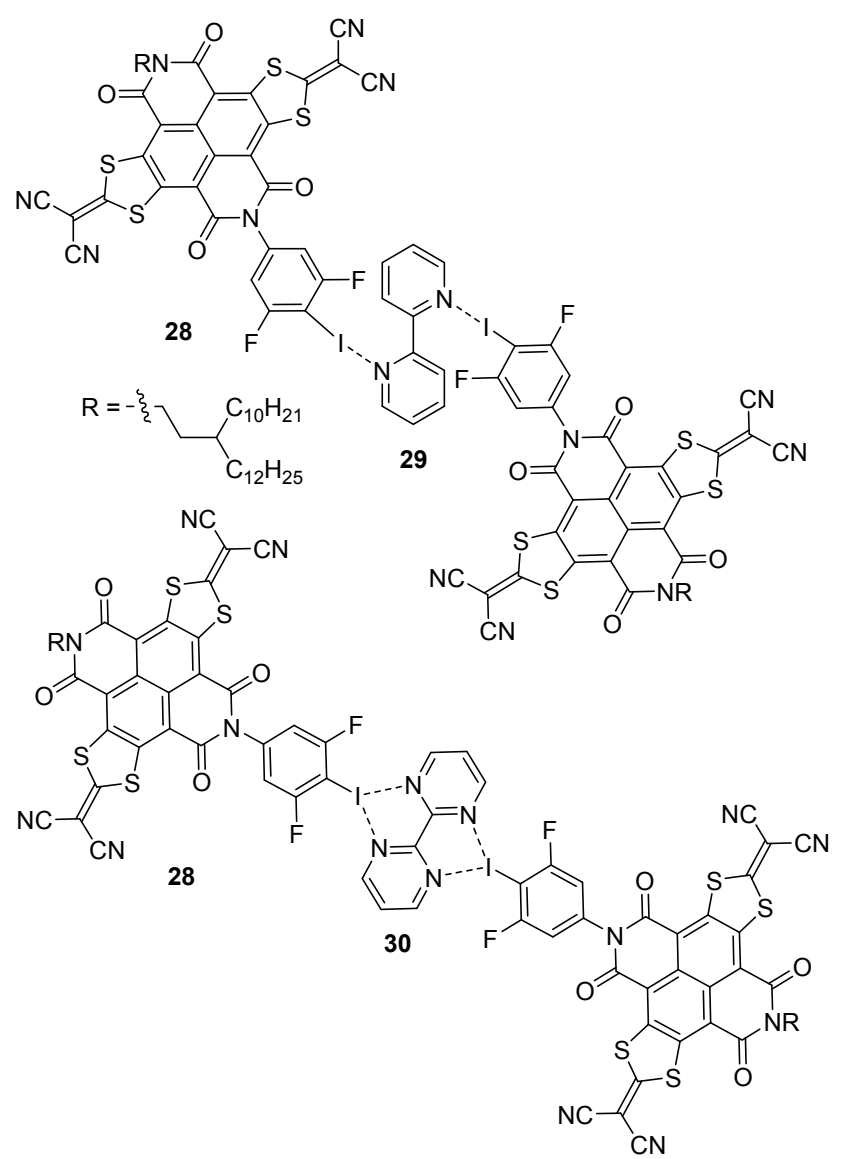

图 13 化合物 28 与 29 和 30 形成的卤键模式

Figure 13 Halogen bonding motif formed between compounds $\mathbf{2 8}$ and $\mathbf{2 9}$ or $\mathbf{3 0}$

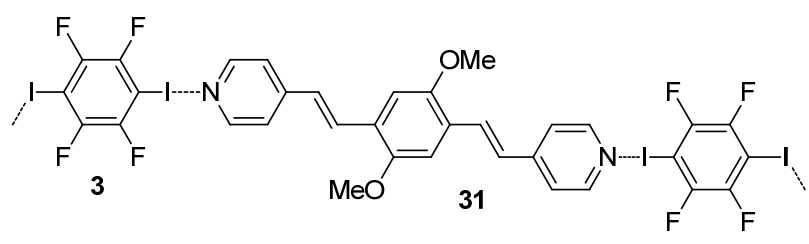

图 14 化合物 3 和 31 形成的卤键模式

Figure 14 Halogen bonding motif formed between compounds $\mathbf{3}$ and $\mathbf{3 1}$

基于卤键的有机功能材料设计，在其它领域也取得 了很多进展. 例如, 姜世梅等 ${ }^{[52]}$ 报道基于芳香亚胺的卤 键诱导的晶体可以产生液晶相. 冯宇等 ${ }^{[53]}$ 报道, 外侧引 入四氟碘苯的树枝状分子凝胶可以与 $\mathrm{Cl}^{-}$形成卤键，从 而实现后者的可视化识别. 晋卫军等 ${ }^{[54]}$ 报道, 三甲基丙 基氯化铵修饰的硅胶可以在固-液界面通过 $\mathrm{C}-\mathrm{I} \cdots \mathrm{Cl}^{-}$ 卤键, 吸附多氟碘苯, 以实现对后者的固相萃取. 这些 不同方面的研究进展都表明了卤键在材料设计方面具 有广泛的潜力.

\section{5 卤键促进的有机反应}

维生素 $\mathrm{K}_{3}$ (甲菜醌, 32) 与 3 或化合物 $33 \sim 35$ 在晶体
中形成 $\mathrm{I} \cdots \mathrm{O}$ 或 $\mathrm{Br} \cdots \mathrm{O}$ 卤键，并由此控制 32 同向堆积(图 15). 梅雪峰等 ${ }^{[55]}$ 利用这一共晶控制 32 的光二聚反应, 以最高 $95 \%$ 的产率形成顺式产物 syn-36. 而纯的 32 固体 的光二聚反应同时生成顺式和反式产物，产率分别为 $57 \%$ 和 $43 \%$. 因此，通过卤键形成共晶显著提高了顺式 产物的选择性.
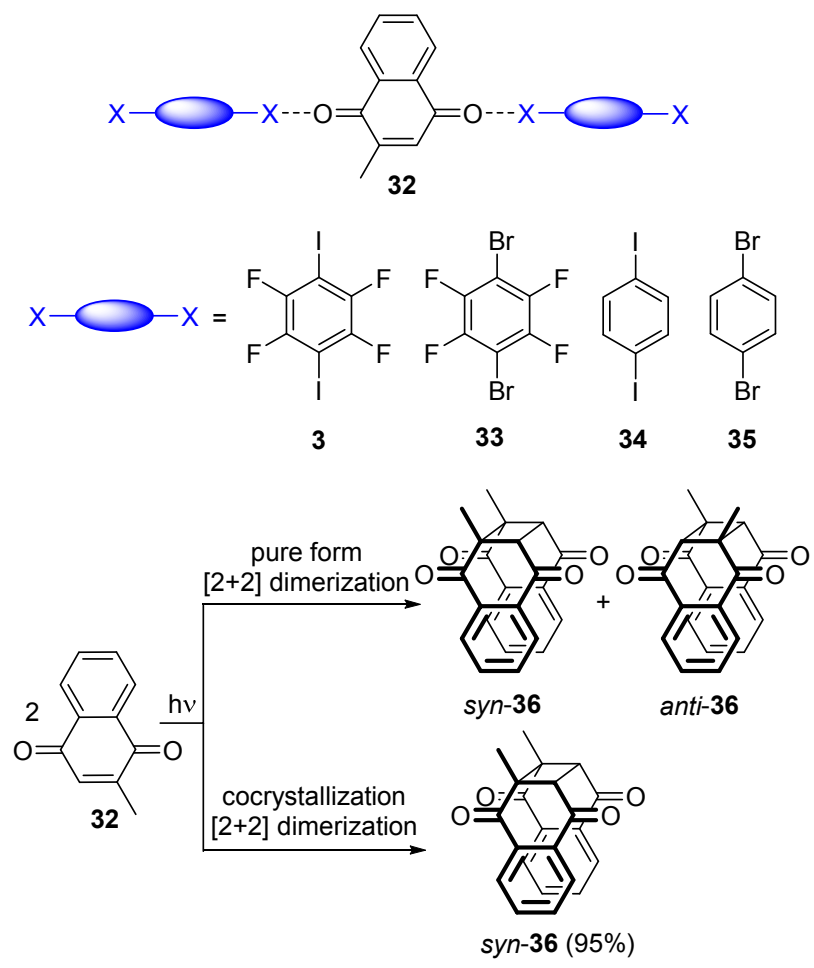

图 15 化合物 32 与 $3,33 \sim 35$ 形成的卤键模式及其纯样品和 共晶内的光二聚反应

Figure 15 Halogen bonding motif formed between $\mathbf{3 2}$ and $\mathbf{3}$ or $33 \sim 35$ and its photodimerization in pure form or in cocrystal to yield conformers 36

陈庆云等 ${ }^{[12 ~ 14]}$ 早期曾研究全氟碘代烷与胺类形成 供体一受体相互作用。成佳佳等 ${ }^{[56]}$ 报道，在全氟碘丁烷 存在下，不加过渡金属，简单加热或可见光照射下，腙 37 或胺 38 可以与醚或硫醚 39 反应，生成相应的缩醛胺 醚腙衍生物 40 或缩醛胺醚 41(图 16). 相应反应被应用 于制备抗病毒和抗肿瘤药物喃氟啶. 化合物 $\mathbf{3 7}$ 和 $\mathbf{3 8}$ 与 全氟丁基碘形成 $\mathrm{N} \cdots \mathrm{I}$ 卤键复合物, 光照或加热使其 $\mathrm{C}$ - $\mathrm{I}$ 键均裂，形成全氟丁基自由基，该自由基算取醚的 $\alpha$-碳上氢原子产生碳自由基, 最终实现 $\mathrm{C}-\mathrm{N}$ 成键反应.

陈弓等 ${ }^{[57]}$ 报道了全氟烷基碘与胺或醚形成卤键复 合物，该卤键复合物在可见光促进下产生全氟烷基自由 基，从而从联苯异腈 42 出发制备 6-氟烷基菲啶 43(图 17). 由此产生的氟烷基自由基还可以发生烯烃加成反 应，制备相应的氟烷基化的碘代物. 与芳烃或吲哚反应 可以实现两类分子的氟烷基化等. 根据芳环结构和取代 基差异，反应产率在 56\% 94\%. 


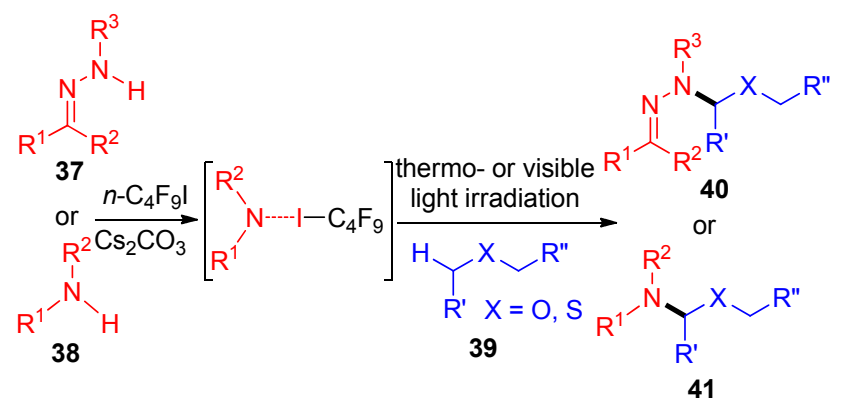

图 16 卤键促进的醚 $\alpha-\mathrm{C}-\mathrm{H}$ 健活化胺化制备缩醛胺醚

Figure 16 Halogen-bond-promoted $\alpha-\mathrm{C}-\mathrm{H}$ amination of ethers for the synthesis of hemiaminal ethers

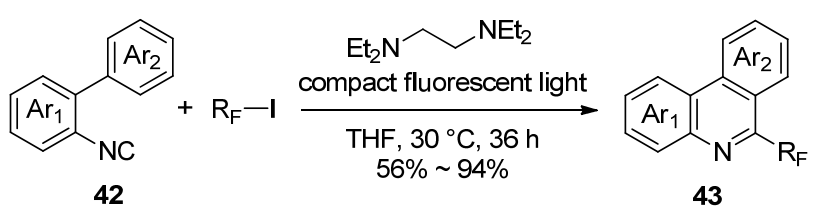

图 17 卤键介质的可见光引发的芳基异腈 $\mathbf{4 2}$ 的全氟烷基加成 反应制备 6-氟烷基菲啶 $\mathbf{4 3}$

Figure 17 Halogen bonding-mediated perfluoroalkyl addition of aromatic isocyanates $\mathbf{4 2}$ to prepare 6-perfluoroalkyl phenanthridines $\mathbf{4 3}$

俞寿云等报道(图 18), 利用全氟烷基碘 45 与三乙烯 二胺 $\mathrm{DABCO}$ 形成卤键可以活化 $\mathrm{C}-\mathrm{I}$ 键. 在可见光照射 下，二异腈 44 可以与 $\mathbf{4 5}$ 反应生成碘代氟烷基化喹喔啉 衍生物 $\mathbf{4 6}^{[58]}$, 而与全氟溴代烷 47 在类似条件下反应, 则只生成相应的氟烷基化产物 $\mathbf{4 8}^{[59]}$. 当用烷基或芳基 炔 49 为底物时, 发生加成反应生成相应碘代全氟烷基 化产物 $\mathbf{5 0}^{[60]}$, 与通过其它方法引发的末端炔烃的反应 结果类似 ${ }^{[61]}$. 利用卤键促进的有机反应研究, 近期多个 研究组也相继取得了新的进展, 成为有机氟化学研究的 活跃领域 ${ }^{[62]}$.

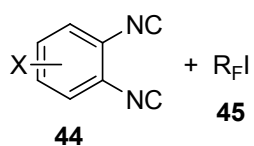

44<smiles>[R17][13C](Br)Br</smiles>

44<smiles></smiles>

49
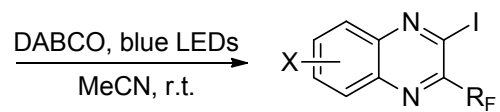

46

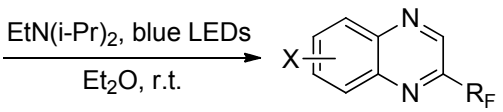

48
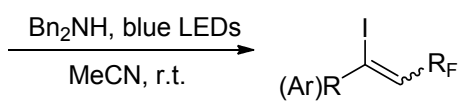

50

图 18 卤键促进的可见光引发的异腈 44 和炔 49 与全氟烷基 碘 45 或全氟烷基溴 47 的反应

Figure 18 Halogen bonding-promoted visible light-initiated reactions of diisocyanates 44 and alkynes 49 with perfluoroalkyl iodides $\mathbf{4 5}$ or bromides $\mathbf{4 7}$

\section{6 结论与展望}

总结了我国学者在有机全氟化合物, 特别是碘代全 氟烷烃和芳烃等形成的卤键研究方面的进展. 早期研究 着重发展基于卤键的晶体工程. 最近的研究越来越重视 卤键在溶液相分子识别，控制有机分子的材料性能和提 高有机反应选择性等方面的应用. 卤键具有显著的方向 性和正交性，易于实现与其它非共价键相互作用的协同 作用，未来在不同领域会获得更多的应用. 就超分子化 学和分子自组装研究来说, 多个卤键的协同作用、有序 多孔结构的构筑、互穿结构的组装和新的结构基元设计 等应获得更多关注. 卤键促进的化学反应近年来进展迅 速，预期在不对称催化或诱导及多组分反应方面会有新 的进展. 卤键已经被证明能在晶体中调控分子间的电子 传输和共晶的发光性能, 利用卤键控制分子在二维和三 维空间的有序排列, 仍需要系统深入的研究.

\section{References}

[1] Needham, P. Studies in History and Philosophy of Science 2013, 44, 51.

[2] Pauling, L. The Nature of the Chemical Bond and the Structure of Molecules and Crystals, 3rd, Ithaca, Cornell University Press, 1960.

[3] Guthrie, F. J. Chem. Soc. 1863, 16, 239.

[4] Hassel, O.; Hvoslef, J.; Vihovde, E. H.; Sörensen, N. A. Acta Chem. Scand. 1954, 8, 873

[5] Dumas, J.-M.; Peurichard, H.; Gomel, M. J. Chem. Res., Synop 1978, 2, 54.

[6] (a) Farina, A.; Meille, S. V.; Messina, M. T.; Metrangolo, P.; Resnati, G.; Vecchio, G. Angew. Chem., Int. Ed. 1999, 38, 2433.

(b) Metrangolo, P.; Resnati, G. Chem. Eur. J. 2001, 7, 2511.

[7] (a) Clark, T.; Hennemann, M.; Murray, J. S.; Politzer, P. J. Mol. Model. 2007, 13, 291.

(b) Politzer, P.; Lane, P.; Concha, M. C.; Ma, Y.; Murray, J. S. J. Mol. Model. 2007, 13, 305.

(c) Wang, H.; Wang, W.; Jin, W. J. Chem. Rev. 2016, 116, 5072.

[8] Desijaru, G. R.; Ho, P. S.; Kloo, L.; Legon, A. C.; Marquardt, R.; Metrangolo, P.; Politzer, P.; Resnati, G.; Rissanen, K. Pure Appl. Chem. 2013, 85, 1711.

[9] Cheetham, N. F.; Pullin, A. D. E. J. Chem. Soc., Chem. Commun. 1965, 418.

[10] Gutman, V. The Donor-Acceptor Approach to Molecular Interaction, Plenum Press, New York, 1978, p. 41.

[11] Chen, Q. Y.; Qiu, Z. M. J. Fluorine Chem. 1986, 31, 301.

[12] Chen, Q.-Y.; Qiu, Z.-M. J. Fluorine Chem. 1987, 35, 79.

[13] Chen, Q.-Y.; He, Y.-B.; Yang, Z.-Y. Chin. J. Org. Chem. 1988, 451 (in Chinese).

(陈庆云，何亚波，杨震宇，有机化学，1988, 451.)

[14] Chen, Q.-Y.; Li, Z.-T.; Zhou, C.-M. J. Chem. Soc., Perkin Trans. 1 $1993,2457$.

[15] Mukherjee, A.; Tothadi, S.; Desiraju, G. R. Acc. Chem. Res. 2014 $47,2514$.

[16] Ding, X.-H.; Ou, C.-J.; Wang, S.; Xie, L.-H.; Lin, J.-Y.; Wang, J.-P.; Huang, W. CrystEngComm 2017, 19, 5504.

[17] Christopherson, J.-C.; Topic, F.; Barrett, C. J.; Friscic, T. Cryst. Growth Des. 2018, 18, 1245.

[18] (a) Ho, P. S. Top. Curr. Chem. 2015, 358, 241.

(b) Lu, Y.; Liu, Y.; Xu, Z.; Li, H.; Liu, H.; Zhu, W. Exp. Opin. Drug Discovery Dev. 2012, 7, 375.

[19] (a) Breugst, M.; von der Heiden, D.; Schmauck, J. Synthesis 2017, 
49, 3224.

(b) Meyer, F.; Dubois, P. CrystEngComm 2013, 15, 3058.

[20] (a) Ding, H.; Lu, Y.; Wu, W.; Liu, H. Chem. Phys. 2014, 441, 30

(b) Guo, X.; An, X.; Li, Q. J. Phys. Chem. A 2015, 119, 3518.

(c) Wang, Y.; Tong, J.; Wu, W.; Xu, Z.; Lu, Y. Int. J. Quantum Chem. 2015, 115, 884

(d) Han, N.; Zeng, Y.; Sun, C.; Li, X.; Sun, Z.; Meng, L. J. Phys. Chem. A 2014, 118, 7058 .

(e) Wu, W.; Lu, Y.; Liu, Y.; Peng, C.; Liu, H. Comput. Theor. Chem. 2014, 1029, 21

[21] (a) Xiao, L.; Wu, Y.; Yu, Z.; Xu, Z.; Li, J.; Liu, Y.; Yao, J.; Fu, H. Chem.-Eur. J. 2018, 24, 1801.

(b) Li, D.; Yang, X.; Yan, D. ACS Appl. Mater. Interfaces 2018, 10, 34377.

(c) Wang, M.; Cheng, C.; Song, J.; Wang, J.; Zhou, X.; Xiang, H.; Liu, J. Chin. J. Chem. 2018, 36, 698 .

[22] (a) Zhang, Q.; Xu, Z.; Zhu, W. J. Chem. Inf. Model. 2017, 57, 22. (b) Lu, Y.; Shi, T.; Wang, Y.; Yang, H.; Yan, X.; Luo, X.; Jiang, H.; Zhu, We. J. Med. Chem. 2009, 52, 2854.

(c) Ren, J.; He, Y.; Chen, W.; Chen, T.; Wang, G.; Wang, Z.; Xu, Z.; Luo, X.; Zhu, W.; Jiang, H.; Shen, J.; Xu, Y. J. Med. Chem. 2014, 57, 3588 .

[23] (a) Chan, Y.-C.; Yeung, Y.-Y. Angew. Chem., Int. Ed. 2018, 57, 3483 .

(b) Jiang, S.; Zhang, L.; Cui, D.; Yao, Z.; Gao, B.; Lin, J.; Wei, D. Sci. Rep. 2016, 6, 34750 .

(c) Guo, F.; Ye, L.; Li, A.; Yang, X.; Yang, C.; Yu, H. Tetrahedron Lett. 2016, 57, 1944.

[24] (a) Yao, Z.-F.; Wang, J.-Y.; Pei, J. Cryst. Growth Des. 2018, $18,7$.

(b) Chen, X.-L.; Zhang, Y.; Zhang, M.-Y.; Zeng, M.-H. Chin. J. Chem. 2017, 35, 927.

(c) Liu, S.-Y.; Zhang, J.-P.; Chen, X.-M. Cryst. Growth Des. 2017, 17,1441 .

(d) Li, B.; Zang, S.-Q.; Wang, L.-Y.; Mak, T. C. W. Coord. Chem. Rev. 2016, 308, 1 .

(e) Moulton, B.; Zaworotko, M. J. Chem. Rev. 2001, 101, 1629.

[25] (a) Saha, S.; Mishra, M. K.; Reddy, C. M.; Desiraju, G. R. Acc. Chem. Res. 2018, 51, 2957.

(b) Liu, H.; Cheng, X.; Bian, Z.; Ye, K.; Zhang, H. Chin. Chem. Lett. 2018, 29, 1537.

(c) Wang, H.-H.; Liu, H.-Y.; Cheng, F.; Ali, A.; Shi, L.; Xiao, X.-Y.; Chang, C.-K. Chin. Chem. Lett. 2018, 29, 1404.

(d) Asghar, M. Adnan; Zhang, J.; Han, S.; Sun, Z.; Ji, C.; Zeb, A.; Luo, J. Chin. Chem. Lett. 2018, 29, 285.

(e) Li, T.; Wei, H.; Zhang, Z.; Zhao, Y.; Sui, Y.; Wang, X. Sci. China Chem. 2017, 60, 602 .

(f) Bernstein, J.; Davis, R. E.; Shimoni, L.; Chang, N.-L. Angew. Chem., Int., Ed. Engl. 1995, 34, 1555.

(h) Desiraju, G. R. Angew. Chem., Int. Ed. 2007, 46, 8342.

[26] (a) Liu, Z.-Q.; Liu, Y.; Chen, Y.; Zhao, W.-Q.; Fang, W.-N. Chin. Chem. Lett. 2017, 28, 297.

(b) Chen, L.-Z.; Cao, X.-X. Chin. Chem. Lett. 2017, 28, 400. (c) Wang, W.-H.; Gao, Q.; Li, A.-L.; Jia, Y.-Y.; Zhang, S.-Y.; Wang, J.-H.; Zhang, Y.-H.; Bu, X.-H. Chin. Chem. Lett. 2018, 29, 336.

(d) Xu, H.; Wu, P. Chin. J. Chem. 2017, 35, 836

(e) Chen, X.-M.; Tong, M.-L. Acc. Chem. Res. 2007, 40, 162.

[27] (a) Meng, D.; Liang, H.; Chen, Q.; Shen, X. Chin. Chem. Lett. 2018, 29, 447 .

(b) Luan, H.; Sun, H.; Xue, B.; Li, X. Chin. J. Org. Chem. 2017, 37, 1392 (in Chinese)

(栾化金金, 孙宏建, 薛本静, 李晓燕, 有机化学, 2017, 37, 1392.)

(c) Zhang, W.; Chen, D.; Liu, X.; Huang, C.; Zhu, B. Chin. J. Org. Chem. 2017, 37, 474 (in Chinese).

(张文龙, 陈冬梅, 刘兴丽, 黄超, 朱必学, 有机化学, 2017, 37, 474.)

(d) Li, C.-P.; Du, M. Chem. Commun. 2011, 47, 5958.

[28] (a) Liu, C.-M.; Zhang, D.-Q.; Hao, X.; Zhu, D.-B. Sci. China Chem. 2017, 60, 358 . (b) Luo, Y.-S.; Chen, J.-L.; Zeng, X.-H.; Qiu, L.; He, L.-H.; Liu, S.-J.; Wen, H.-R. Chin. Chem. Lett. 2017, 28, 1027.

(c) Deng, C.-D.; Qiao, Y.-J.; Chen, Q.-D.; Shen, X.-H. Chin. Chem. Lett. 2017, 28, 19.

(d) Peng, R.; Li, M.; Li, D. Coord. Chem. Rev. 2010, 254, 1

[29] (a) Aakeröy, C. B.; Spartz, C. L. Top. Curr. Chem. 2015, 358, 155.

(b) Berger, G.; Soubhye, J.; Meyer, F. Polym. Chem. 2015, 6, 3559 .

(c) Pang, X.; Jin, W. J. Top. Curr. Chem. 2015, 359, 115.

[30] Voth, A. R.; Khuu, P.; Oishi, K.; Ho, P. S. Nat. Chem. 2009, 1, 74.

[31] Chu, Q.; Wang, Z.; Huang, Q.; Yan, C.; Zhu, S. J. Am. Chem. Soc. 2001, 123, 11069 .

[32] (a) Chu, Q.; Wang, Z.; Huang, Q.; Yan, C.; Zhu, S. New J. Chem 2003, 27, 1522 .

(b) Zhu, S.; Xing, C.; Xu, W.; Li, Z. Tetrahedron Lett. 2004, 45, 777.

[33] Ji, B.; Wang, W.; Deng, D.; Zhang, Y. Cryst. Growth Des. 2011, 11,3622 .

[34] (a) Yan, D.; Bučar, D.-K.; Delori, A.; Patel, B.; Lloyd, G. O.; Jones, W.; Duan, X. Chem.-Eur. J. 2013, 19, 8213.

(b) Yan, D.; Yang, H.; Meng, Q.; Lin, H.; Wei, M. Adv. Funct. Mater. 2014, 24, 587.

(c) Lin, H.; Chang, X.; Yan, D.; Fang, W.-H.; Cui, G. Chem. Sci. 2017, 8, 2086.

(d) Yan, D.; Evans, D. G. Mater. Horiz. 2014, 1, 46

(e) Li, S.; Lin, Y.; Yan, D. J. Mater. Chem. C 2016, 4, 2527.

(f) Fan, G.; Yan, D. Adv. Opt. Mater. 2016, 4, 2139.

(g) Yan, D. Chem.-Eur. J. 2015, 21, 4880.

[35] Pang, X.; Zhao, X. R.; Wang, H.; Sun, H.-L.; Jin, W. J. Cryst. Growth Des. 2013, 13, 3739

[36] Liu, P.; Li, Z.; Shi, B.; Liu, J.; Zhu, H.; Huang, F. Chem.-Eur. J. 2018, 24, 4264.

[37] Han, C.; Zhao, D.; Dong, S. Chem. Commun. 2018, 54, 13099.

[38] Politzer, P.; Murray, J. S.; Clark, T. Phys. Chem. Chem. Phys. 2010, 12, 7748.

[39] (a) Gao, H. Y.; Zhao, X. R.; Wang, H.; Pang, X.; Jin, W. J. Cryst. Growth Des. 2012, 12, 4377.

(b) Shen, Q. J.; Pang, X.; Zhao, X. R.; Gao, H. Y.; Sun, H.-L.; Jin, W. J. CrystEngComm 2012, 14, 5027.

(c) Gao, H.; Zhao, X.; Wang, H.; Pang, X.; Jin, W. Chin. J. Chem. 2013, 31, 1279.

[40] Liu, C.-Z.; Koppireddi, S.; Wang, H.; Zhang, D.-W.; Li, Z.-T. Angew. Chem., Int. Ed. 2019, 58, 226.

[41] (a) Yang, Y.; Wang, C. Chem. Soc. Rev. 2009, 38, 2576.

(b) Wang, D.; Wan, L.-J.; Bai, C.-L. Mater. Sci. Eng., R 2010, 70, 169.

(c) Jin, X.; Cramer, J. R.; Chen, Q.-W.; Liang, H.-L.; Shang, J.; Shao, X.; Chen, W.; Xu, G.-Q.; Gothelf, K. V.; Wu, K. Chin. Chem. Lett. 2017, 28, 525

[42] (a) Borges, J.; Mano, J. F. Chem. Rev. 2014, 114, 8883. (b) Xu, H.; Schönhoff, M.; Zhang, X. Small 2012, 8, 517.

[43] Zheng, Q.-N.; Liu, X.-H.; Chen, T.; Yan, H.-J.; Cook, T.; Wang, D.; Stang, P. S.; Wan, L.-J. J. Am. Chem. Soc. 2015, 137, 6128.

[44] (a) Yang, X.; Wang, F.; Chen, Q.; Wang, L.; Wang, Z. Chin. Sci. Bull. 2007, 52, 1856.

(b) Wang, F.; Ma, N.; Chen, Q.; Wang, W.; Wang, L. Langmuir 2007, 23, 9540 .

[45] Beale, T. M.; Chudzinski, M. G.; Sarwar, M. G.; Taylor, M. S. Chem. Soc. Rev. 2013, 42, 1667.

[46] (a) Zhu, Y.-Y.; Wang, G.-T.; Wang, R.-X.; Li, Z.-T. Cryst. Growth Des. 2009, 9, 4778.

(b) You, L.-Y.; Chen, S.-G.; Zhao, X.; Liu, Y.; Lan, W.-Y.; Zhang, Y.; Lu, H.-J.; Cao, C.-Y.; Li, Z.-T. Angew. Chem., Int. Ed. 2012, 51, 1657.

(c) Sun, G.; Nie, C.; Zhao, X.; Li, Z. Chin. J. Org. Chem. 2017, 37, 1757 (in Chinese)

(孙广军, 聂承斌, 赵新, 黎占亭, 有机化学, 2017, 37, 1757.)

(d) Wang, D.-Y.; You, L.-Y.; Wang, J.-L.; Wang, H.; Zhang, D.-W.; 
Li, Z.-T. Tetrahedron Lett. 2013, 54, 6967.

[47] (a) Gao, H. Y.; Shen, Q. J.; Zhao, X. R.; Yan, X. Y.; Pang, X.; Jin, W. J. J. Mater. Chem. 2012, 22, 5336.

(b) Liu, R.; Wang, H.; Jin, W. J. Cryst. Growth Des. 2017, 17, 3331.

(c) Wu, W. X.; Wang, H.; Jin, W. J. Cryst. Growth Des. 2018, 18, 6742 .

[48] Sun, H.; Wang, M.; Khan, A.; Shan, Y.; Zhao, K.; Usman, R.; Xu, C. ChemistrySelect 2017, 2, 6323.

[49] Zhu, W.; Zheng, R.; Zhen, Y.; Yu, Z.; Dong, H.; Fu, H.; Shi, Q.; Hu, W. J. Am. Chem. Soc. 2015, 137, 11038 .

[50] Li, J.; Hu, Y.-H.; Ge, C.-W.; Gong, H.-G.; Gao, X.-K. Chin. Chem. Lett. 2018, 29, 423.

[51] Zhuo, M.-P.; Tao, Y.-C.; Wang, X.-D.; Wu, Y.; Chen, S.; Liao, L.-S.; Jiang, L. Angew. Chem., Int. Ed. 2018, 57, 11300.

[52] Wang, Y.; Shang, H.; Li, B.; Zhang, H.; Jiang, S. CrystEngComm 2017, 19, 3801.

[53] Liu, Z.-X.; Sun, Y.; Feng, Y.; Chen, H.; He, Y.-M.; Fan, Q.-H. Chem. Commun. 2016, 52, 2269.

[54] Li, C.; Li, L.; Yang, X.; Jin, W. J. Colloids Surf., A 2017, 520, 497.

[55] Zhu, B.; Wang, J.-R.; Zhang, Q.; Mei, X. CrystEngComm 2016, 18 , 6327.

[56] Pan, Z.; Fan, Z.; Lu, B.; Cheng, J. Adv. Synth. Catal. 2018, 360, 1761.

[57] Wang, Y.; Wang, J.; Li, G.-X.; He, G.; Chen, G. Org. Lett. 2017,
$19,1442$.

[58] Sun, X.; Wang, W.; Li, Y.; Ma, J.; Yu, S. Org. Lett. 2016, 18, 4638.

[59] Sun, X.; Wang, W.; Ma, J.; Yu, S. Acta Chim. Sinica 2017, 75, 115 (in Chinese). (孙晓阳，王文敏，马晶，俞寿云，化学学报 2017, 75, 115.)

[60] Sun, X.; He, Y.; Yu, S. J. Photochem. Photobiol. A Chem. 2018, $355,326$.

[61] (a) Chen, Q.; Qiu, Z.; Yang, Z. J. Fluorine Chem. 1987, 36, 149.

(b) Huang, W.; Lü, L.; Zhang, Y. Chin. J. Chem. 1990, 350

(c) Huang, W.; Xie, Y. Chin. Chem. Lett. 1990, 1, 165.

(d) Huang, W.; Lü, Chin. J. Chem. 1992, 10, 268.

(e) Zhang, C.-P.; Chen, Q.-Y.; Guo, Y.; Xiao, J.-C.; Gu, Y.-C. Chem. Soc. Rev. 2012, 41, 4536.

[62] (a) Li, Y.; Lu, Y.; Mao, R.; Li, Z.; Wu, J. Org. Chem. Front. 2017, 4, 1745.

(b) Chen, T.; Guo, Y.; Sun, K.; Wu, L.-Z.; Liu, W.-Q.; Liu, C.; Huang, Y.; Chen, Q.-Y. Org. Chem. Front. 2018, 5, 1045.

(c) Wang, R.; Guan, W.; Han, Z.-B.; Liang, F.; Suga, T.; Bi, X.; Nishide, H. Org. Lett. 2017, 19, 2358.

(d) Huang, Y.; Lei, Y.-Y.; Zhao, L.; Gu, J.; Yao, Q.; Wang, Z.; Li, X.-F.; Zhang, X.; He, C.-Y. Chem. Commun. 2018, 54, 13662

(e) Li, M.; Wang, C.-T.; Qiu, Y.-F.; Zhu, X.-Y.; Han, Y.-P.; Xia, Y.; Li, X.-S.; Liang, Y.-M. Chem. Commun. 2018, 54, 5334.

(f) Guo, Q.; Wang, M.; Liu, H.; Wang, R.; Xu, Z. Angew. Chem., Int. Ed. 2018, 57, 4747. 